\title{
Resposta radiodosimétrica de implantes de sementes de biovidros radioativos no cérebro de coelhos
}

\author{
Costa, I.T.; Campos, T.P.R.;
}

Programa de Pós-Graduação em Ciências e Técnicas Nucleares - Universidade Federal de Minas Gerais. Av. Antônio Carlos, 6627, prédio PCA-1, CEP - 31270901, BH, MG, Brasil e-mail: itemponi@yahoo.com.br; campos@ nuclear.ufmg.br

\section{RESUMO}

Implantes intersticiais de sementes radioativas são empregados como uma forma eficiente de tratamento de tumores cerebrais. Os biovidros surgiram como uma interessante alternativa aos materiais metálicos implantados por poderem ser absorvidos pelo organismo, diminuindo as possibilidades de efeitos colaterais. $\mathrm{O}$ presente trabalho investiga a radiodosimetria de implantes produzidos em cobaias no grupo de pesquisa NRI/UFMG - Núcleo de Radiações Ionizantes. A distribuição espacial da energia específica depositada por unidade de massa, gerada pelas sementes de Sm-153, foi avaliada. Um modelo computacional da região do cérebro foi construído utilizando o software SISCODES produzido pelo grupo de pesquisa NRI. Foram digitalizadas as seções de tomografia computadorizada obtidas dos coelhos com implantes de sementes. Estas foram convertidas em um modelo tridimensional de voxel, cujos tecidos, sua composição química e densidade foram identificados. A simulação do transporte de partículas nucleares foi realizada pelo código estocástico MCNP5. Os implantes executados consistiram de 15 sementes cerâmicas de Ca-Si-Sm de $1.6 \mathrm{~mm}$ de comprimento e $0,3 \mathrm{~mm}$ de diâmetro, enriquecidas com Sm-153. Foi previsto no modelo o posicionamento de três filetes com 5 sementes cada, sendo o espaço entre sementes de $1.2 \mathrm{~mm}$ cada e entre filetes de $8 \mathrm{~mm}$. As atividades assumidas para as sementes foram de $120 \mathrm{MBq} / \mathrm{semente}$. Os resultados mostram regiões de isodose projetadas sobre o modelo de voxel dos coelhos, reproduzindo a distribuição espacial da energia depositada por unidade de massa (dose absorvida) no cérebro das cobaias. Foi previsto que a dose absorvida de 3,2Gy produzidas por 15 sementes não é suficiente para o controle tumoral no modelo em estudo. Recomenda-se o aumento do número de sementes, a atividade específica, e redução do afastamento das sementes para 5-6mm aproveitando das emissões betas emitidas do Sm-153.

Palavras chaves: Biovidros, braquiterapia, dosimetria.

\section{Dosimetric response of radioactive bioglass seeds implants on rabbit's brain}

\section{ABSTRACT}

Intersticial implants of radioactive seeds are used as an efficient way of treating brain tumors. Bioglasses is an interesting alternative to the metallic implanted materials, because they can be absorved by the organism, reducing the possibilities of side effects. The present paper investigates the dosimetry by the implants performed on rabbit's brain on the NRI/UFMG research group. The spatial distribution of the specific ionizing energy deposited per unit of mass generated by Sm-153 seeds were evaluated. A computational model of the brain's region was built using the software SISCODES produced by the research group. The sections of the computer tomography of a rabbit, which was included on the experiment, were digitalized. Those were converted in a three dimensional voxel model, including the tissues, its chemical composition and density. A simulation of the particles transport is performed by the estocastic code MCNP5. The implants consist of 15 ceramic Ca-Si-Sm seeds enriched with Sm-153, with $1.6 \mathrm{~mm}$ of length and $0.3 \mathrm{~mm}$ diameter, implanted on the rabbit's brain. It was predicted on the model three ribbons of 5 seeds each, spaced by $1.2 \mathrm{~mm}$, since the ribbons were in a triangular topology whose vertices were spaced by $8 \mathrm{~mm}$. The activities were $120 \mathrm{MBq} / \mathrm{seed}$. The results show isodose regions superposed over the rabbits' model, reproducing the spatial energy deposition on the brain region. The absorbed dose predicted was $3.2 \mathrm{~Gy}$ per 15 
seed; however it was not enough to tumor control. The authors suggest to increase the number of seeds and activity, reduction of the space to 5-6mm among ribbons, improving dose with the beta emitting.

Keywords: Bioglass, brachytherapy, dosimetry.

\section{INTRODUÇÃO}

O câncer se desenvolve quando células de um organismo começam a se multiplicar desordenadamente. Este descontrole é desenvolvido por falhas no DNA celular. Diversas podem ser as causas destas falhas assim como da ineficiência do organismo em repará-las. Em muitos casos, o crescimento anormal do tecido canceroso prejudica o funcionamento dos tecidos e órgãos adjacentes podendo levar o indivíduo ao óbito [2]. Parkin e colaboradores (1999) [12] estimaram para o ano de 2000 que o número de casos novos de câncer em todo o mundo seria superior a 10 milhões; dentre os quais, 53\% ocorreriam nos países em desenvolvimento [12]. No Brasil, as estimativas para o ano de 2006 apontam para 472.050 novos casos de câncer, sendo que este será a terceira maior causa de óbitos no país [8].

Existem mais de 100 doenças caracterizadas como cânceres, sendo incluídos os tumores cerebrais. Os tumores cerebrais podem ser classificados como primários, quando a origem histológica das células cancerosas ocorre no próprio cérebro, e metastáticos, quando as células mutantes se originam em outro local do organismo e são transportadas pela corrente sanguínea ou pelo sistema linfático sendo infiltradas no cérebro. Tumores cerebrais primários ocorrem em pessoas de todas as idades, já os metastáticos são muito mais comuns em adultos. Em termos mundiais estatísticas exatas quanto à ocorrência e evolução de tumores cerebrais não estão disponíveis, porém, em 1990 estimou-se que estes tipos de tumores tenham sido responsável por $1,5 \%$ dos casos diagnosticados e por $2,0 \%$ dos casos de morte por câncer. [1, 2, 18]. Existem atualmente algumas possibilidades de abordagem para o tratamento de um tumor cerebral e elas incluem a intervenção cirúrgica, que oferece grandes riscos de complicações [15], a imunoterapia, a quimioterapia e a radioterapia, sendo que todas as três têm seus respectivos benefícios e efeitos deletérios.

A forma tradicional de radioterapia é a teleterapia sendo que, nas situações em que o tumor é radio sensível, feixes externos de raios $\mathrm{X}$, gama e prótons são direcionados para a região do tumor. A braquiterapia é uma forma alternativa de radioterapia em que implantes intersticiais de material radioativo são incorporados no leito do tumor. Esta técnica, já bastante utilizada atualmente, apresenta várias vantagens sobre a teleterapia, pois, altas doses de radiação podem ser entregues diretamente no leito do tumor. As emissões gamas de energias entre $20-100 \mathrm{keV}$ são pouco penetrante nos tecidos adjacentes, poupando as regiões vizinhas. Assim, sementes de I-125 e Pd-103 mostraram-se adequadas mesmo em casos de tumores reincidentes que, supostamente, são casos mais graves $[2, \underline{7}, \underline{15}]$. A braquiterapia intersticial faz uso da ultrasonografia para guiar e monitorar os implantes e dedicados softwares para avaliação do planejamento radiodosimétrico. Implantes braquiterápicos podem utilizar diversos radioisótopos como ${ }^{137} \mathrm{Cs},{ }^{192} \mathrm{Ir}$, ${ }^{125} \mathrm{I}$, e

${ }^{103} \mathrm{Pd}$ incorpoado em estruturas metálicas. Sementes metálicas de ${ }^{125} \mathrm{I}$ têm sido utilizadas em implantes cerebrais [6]. Estes implantes podem ser permanentes, quando as sementes não são retiradas do organismo, ou no caso contrário, temporários $[6,3]$. Os materiais usados atualmente como fontes na braquiterapia podem ser revestidos por materiais biologicamente inativos. A resposta dos tecidos a estes tipos de material é a formação de uma cápsula fibrosa de espessura variável [5]. Uma extensa fibrose pode acarretar em efeitos colaterais após os implantes. Este fato, aliado ao elevado preço de fabricação deste tipo de fonte [5], torna o uso de sementes de biomateriais, propostos no grupo de pesquisa NRI/CNPq, uma interessante alternativa para a realização deste tipo de procedimento. $[\underline{13}, \underline{14}, \underline{9}, \underline{17}]$ Os implantes com material bioativo são projetados para se degradarem gradualmente e serem substituídos por tecidos locais com o tempo [ $\underline{5}$ ]. A distribuição de dose (dosimetria) do volume a ser tratado pode ser utilizada para avaliar a eficiência do tratamento através da determinação do volume coberto com a dose prescrita. A dosimetria pode ser usada para refinar o tratamento evitando-se ou reduzindo-se a dose em tecidos normais considerados susceptíveis aos efeitos da radiação [11]. O presente trabalho tem o intuito de fazer uma avaliação dosimétrica de implantes de biovidros em modelo animal. Esta análise servirá como base para a avaliação da viabilidade do uso destas fontes bioativas como uma alternativa para o tratamento de tumores cerebrais pela braquiterapia.

\section{MATERIAIS E MÉTODOS}

\section{$2.1 \quad$ Fontes}

O elemento a ser usado como radioemissor é o Samário-153 (Sm-153). Este radionuclídeo já é usado em medicina nuclear para terapias tumorais e paliativos de dores ósseas, devido à suas energias de betas absorvidas localmente, emitidas por desintegração, e também pela sua relativa meia-vida de 46,3 h e o 
seu seletivo metabolismo em ossos [16]. O Sm-153 possui múltiplas emissões de raios beta e emissões gama. A probabilidade de emissão varia para as betas de energia média entre 200 a $265 \mathrm{keV}$ e suas principais gamas são de $103 \mathrm{keV}$ e $69 \mathrm{keV}$. A meia-vida curta do Samário-153 é também de grande relevância para a braquiterapia permanente (aproximadamente de 46, 27 horas). [16]

As fontes, ou sementes bioceramicas, utilizadas no procedimento são fabricadas com os seguintes compostos em mistura: água deionizada, nitrato de samário hexahidratado $\left(\mathrm{Sm}\left(\mathrm{NO}_{3}\right)_{3} \cdot 6 \mathrm{H}_{2} \mathrm{O}\right)$, solução de ácido nítrico $2 \mathrm{~N}\left(\mathrm{HNO}_{3}\right)$, que foi utilizado como catalisador, tetraetilortosilicato $\left(\mathrm{C}_{8} \mathrm{H}_{20} \mathrm{O}_{4} \mathrm{Si}\right)$, também conhecido como TEOS e nitrato de cálcio $\left(\mathrm{Ca}\left(\mathrm{NO}_{3}\right)_{2} \cdot 4 \mathrm{H}_{2} \mathrm{O}\right)$. Após passarem pelo processo Sol-Gel se tornam uma biocerâmica homogênea com $20 \%$ de samário, $30 \%$ de cálcio e $50 \%$ de silício, de formato cilíndrico, com dimensões de aproximadamente $0.3 \times 1.6 \mathrm{~mm}$ e peso em massa de aproximadamente de $2.0 \times 10^{-4} \mathrm{~g}$.

\subsection{Simulação}

A técnica de simulação de Monte Carlo [4] tornou-se uma ferramenta inestimável na dosimetria de fontes para braquiterapia nos últimos anos. Muitos pesquisadores usam atualmente diversos códigos de Monte Carlo para a dosimetria no campo da braquiterapia [19]. Foi utilizado no presente trabalho o Monte Carlo N-Particle (MCNP versão 5). Este código computacional pode ser usado para várias aplicações nucleares. Este código utiliza o método de cálculo de Monte Carlo e simula o transporte de partículas em várias geometrias complexas. Por estas propriedades ele pode ser utilizado para determinar as doses absorvidas em órgãos e tecidos em diversos tipos de procedimentos braquiterápicos [4, 20].

O código MCNP é configurado com entradas que são usadas para criar o modelo geométrico e material. Primeiramente são inseridas as entradas referentes à configuração das áreas de interesse. Este procedimento é importante para a determinação da região na qual o usuário deseja calcular a quantidade de interesse (i.e. deposição de energia, fluência). Em seguida são definidas as superfícies que formam os contornos destas regiões. O código pode modelar planos bidimensionais que podem ser usados para delimitar superfícies tridimensionais com dimensões finitas, como cilindros.

As áreas de interesse são obtidas a partir de tomografias computadorizadas. $\mathrm{O}$ animal utilizado no procedimento foi um coelho da raça Nova Zelândia, macho, pesando aproximadamente $2,5 \mathrm{Kg}$. As tomografias computadorizadas são digitalizadas por processos convencionais. As imagens digitais são então tratadas no software SISCODES, desenvolvido pelo próprio grupo de pesquisa. Este software é utilizado para tornar possível o reconhecimento das regiões de interesse pelo código MCNP5. Com ele as tomografias são transformadas num mapa de voxels. Regiões correspondentes a órgãos e tecidos específicos são delimitadas e recebem cores específicas. Estas cores utilizadas fazem referência a um banco de dados com a composição química e densidade de diversos tecidos do animal em questão. Os cortes tomográficos são tratados um a um e então montados como um modelo de voxels tridimensional. Comparações entre as tomografias dos coelhos e suas correspondentes seções tratadas no SISCODES podem ser vistas na Figura 1.
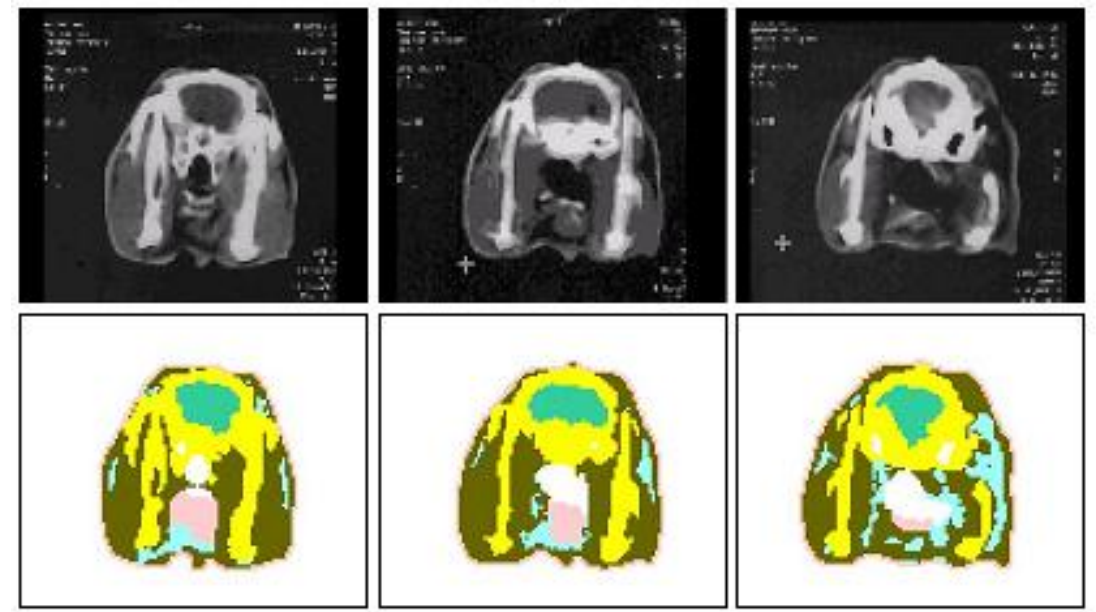

Figura 1: Comparação das tomografias originais e respectivas seções tratadas no SISCODES.

O modelo de voxel foi desenvolvido é definida uma região de interesse para as avaliações de dose. No presente trabalho esta região foi representada pelo cérebro do coelho modelado. Os dados correspondentes aos tecidos presentes em cada voxel, assim como a região de interesse, são então exportados para o MCNP5. 
O contorno fora da região do modelo utilizada no MCNP5 é representado por uma topologia que tem o formato geométrico referente ao corpo de coelho. A digitalização total do corpo se mostra desnecessária e uma aproximação topológica construída por seções de uma elipse delimitada por planos foi utilizada como base. A este volume é introduzido o modelo de vóxel em três dimensões criado anteriormente. A Figura 2 mostra tanto o volume delimitador quanto o modelo gerado e importado do SISCODES.

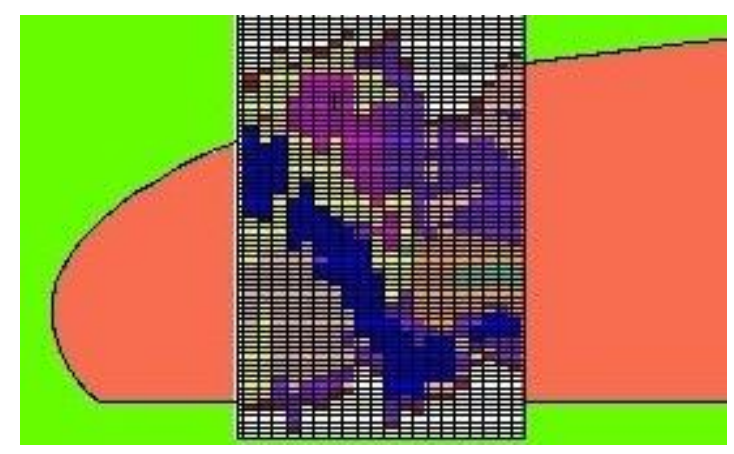

Figura 2: Em laranja está a região delimitadora representando o corpo do coelho. Em diversas cores está a região de interesse para cálculos.

Após a construção do modelo, são inseridas as fontes de biovidros em seus locais específicos. Os três segmentos de implantes, ditos filetes, podem ser vistos nas Figs.3, 4 e 5.

Nesta simulação foram simulados 3 filetes como pode ser observado nos cortes apresentados nas Figs. 3 e 4. Cada filete contém 5 sementes espaçadas de $1.2 \mathrm{~mm}$, sendo que o espaçamento entre filetes foi de $8 \mathrm{~mm}$, compondo um triangulo eqüilátero.

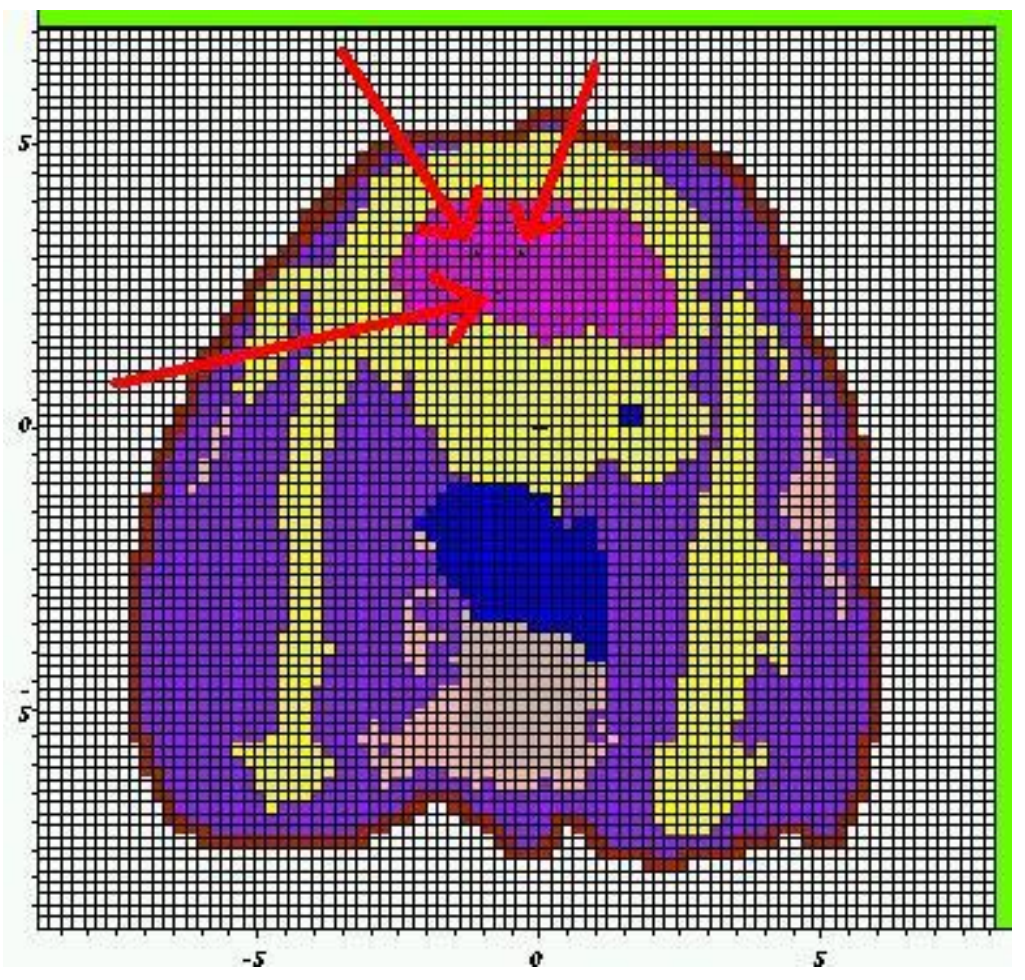

Figura 3: Corte transversal da região de implante. Setas vermelhas foram adicionadas para melhor visualização das sementes. 


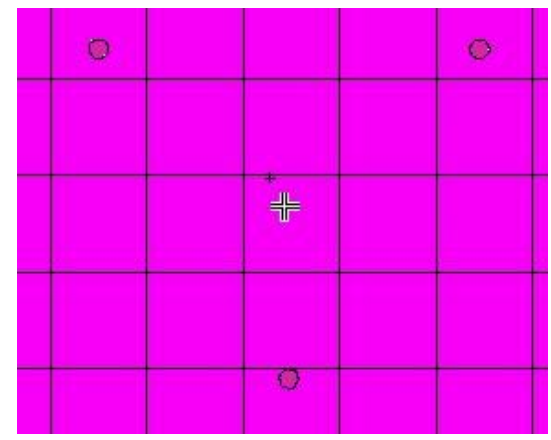

Figura 4: Corte transversal ampliado da região implantada.

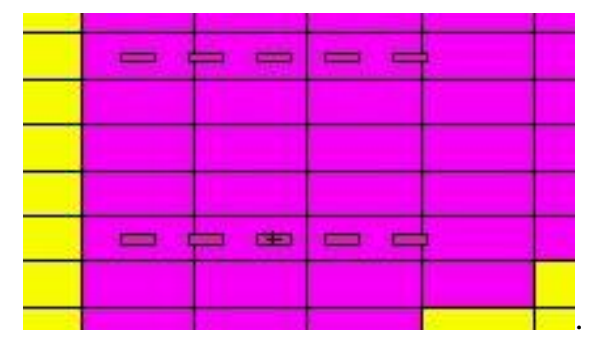

Figura 5: Corte longitudinal ampliado da região implantada.

O Samário-153 decai por emissão $\beta^{-}$em Európio-153. Este nuclídeo filho, ao ir para seu estado de energia fundamental emite também raios gamas. A radiação $\beta$ emitida neste decaimento tem penetração relativamente pequena, em torno de $2-3 \mathrm{~mm}$. No caso de implantes cerebrais, sua energia seria toda depositada junto à própria fonte, no tumor. Por este motivo se torna difícil fazer a distribuição espacial da energia das betas no modelo visto a resolução dos vóxels de 1x1x5mm. Assim, apenas a distribuição espacial das doses absorvidas devido a emissão gama do Sm-153 será avaliada no método adotado.

\section{RESULTADOS}

O volume estudado foi montado a partir de 8 cortes tomográficos. Depois de finalizado o processo de simulação, o valor da dose recebida para cada região do volume pode ser vista projetada sob as seções do próprio modelo computacional de voxel. Na Figura 6 é possível ver os resultados da simulação efetuada pelo MCNP5.

Os dados de saída da simulação efetuada com o código MCNP5 são visibilizados como regiões de isodose plotadas sobre as seções do modelo de voxel, apenas sombreadas. Cada cor representa uma intensidade relativa à dose máxima absorvida. Em vermelho estão as regiões com absorção de $80 \%-100 \%$ da dose máxima, em laranja a absorção é de 50\% - 80\%, em amarelo 10\% - 50\%, em verde 5\% - 10\% e, em azul, $1 \%-5 \%$ desta dose.

A taxa de dose máxima atingida na região estudada foi $2,64 \times 10^{-5} \mathrm{~Gy} \mathrm{~h}^{-1} \mathrm{MBq}^{-1}$.

A partir deste dado temos que a dose acumulada total, obtida através da equação 1 será igual a $1,76 \times 10^{-3} \mathrm{~Gy} \mathrm{MBq}^{-1}$.

$$
D_{\max }\left[G y M B q^{-1}\right]=1,44 . T_{1 / 2} . T D M\left[G y h^{-1} M B q^{-1}\right]
$$

sendo TDM a taxa de dose máxima obtida na simulação.

Tendo cada semente a atividade de $120 \mathrm{MBq}$, e tendo sido feito um implante de 15 sementes, avaliase que a atividade total do implante será de 1,8 GBq. Assim produzir-se-á uma dose total no implante de aproximadamente $3,2 \mathrm{~Gy}$.

Em um tratamento convencional de braquiterapia utiliza-se, geralmente, mais de 100 sementes num único implante. Com esta quantidade de fontes, a dose obtida no procedimento seria compatível com os implantes de sementes de I-125 atualmente realizados.

Através da análise dos resultados, pode-se perceber que em praticamente nenhum momento uma dose maior que $5 \%$ da dose máxima foi detectada fora da região de interesse. 


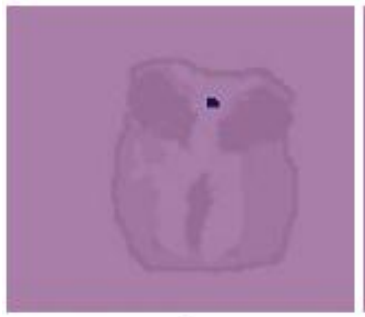

1

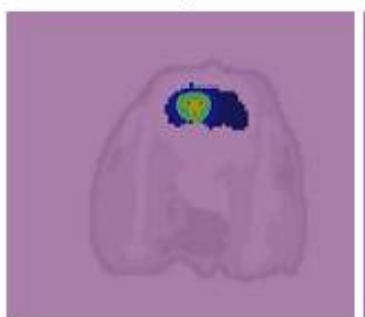

5

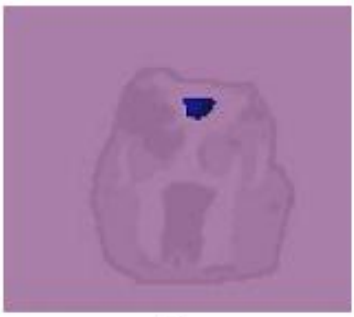

2

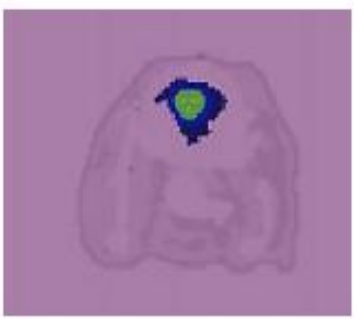

6

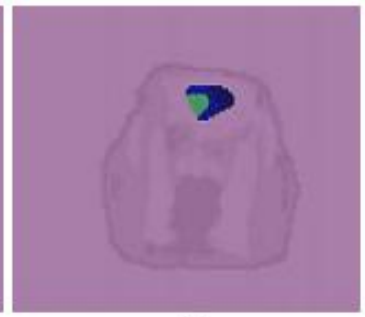

3

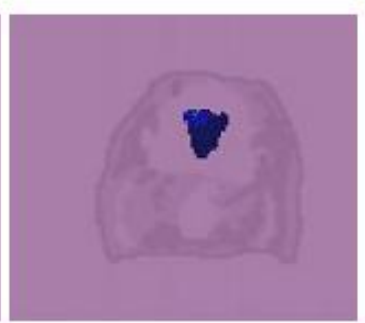

7

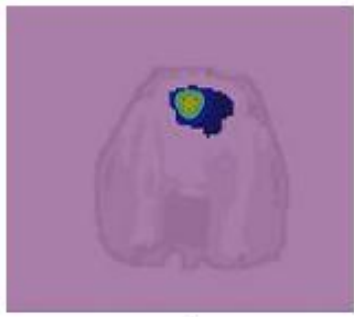

4

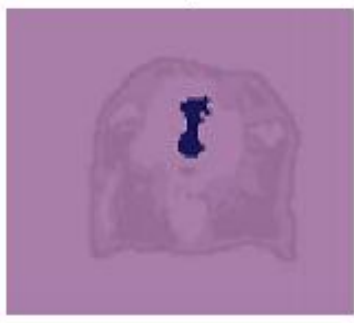

8

Figura 6: Dados de saída da simulação com o MCNP5. As figuras mostram regiões de isodose onde os valores em relação à dose máxima conseguida variam conforme as cores.

\section{CONCLUSÃO}

A simulação realizada no presente trabalho se mostrou adequada para predizer a dose de implantes no cérebro de coelhos, podendo ser utilizada para futuras avaliações de controle tumoral. Todo o processo, desde a aquisição das imagens tomográficas até a obtenção da distribuição de dose, passando pela montagem do modelo computacional e do tratamento de dados no código MCNP5 pôde ser realizado.

Os dados obtidos após a simulação mostram a viabilidade dosimétrica dos implantes. Pode ser facilmente visualizado nas imagens obtidas pelo método de Monte Carlo que a dose entregue pelas fontes será depositado quase que integralmente na região de interesse, no caso, o cérebro da cobaia.

A dose preconizada para 15 sementes implantadas foi de 3,2 Gy, insuficiente para o controle tumoral. Para trabalhos futuros de controle tumoral por implantes de sementes, pretende-se introduzir maior numero de sementes e mesmo desenvolver implantes com filetes espaçados de 5-6 mm, permitindo uma maior dose no centro dos implantes com a contribuição das emissões betas. Dosimetria beta também deve ser incluída futuramente nos resultados.

A presente pesquisa está em desenvolvimento no grupo de pesquisa NRI/PCTN. Avaliações experimentais de implantes radioativos em cobaias, com tumor induzido, estão em progresso. Além das sementes testadas neste trabalho, outros radioisótopos emissores betas estão sendo avaliados.

\section{BIBLIOGRAFIA}

[1] American Brain Tumor Association, http://neurosurgery.mgh.harvard.edu/abta/primer.htm\#Section1, $A$ Primer of Brain Tumors, Acessado em Abril 2006

[2] BETHESDA, M., Adult brain tumor, National Cancer Institute, www.cancer.gov, Acessado em Setembro de 2005.

[3] BLUE, T.E., "Description and analysis of an accelerator-based production method of the brachytherapy source", Nuclear Technology, v. 82, pp. 304-310, 1988

[4] BRIESMEISTER, J.F., MCNP-A General Monte Carlo N-Particle Transport Code, version 4C, Los Alamos National Laboratories, Los Alamos, NM, 2000.

[5] CAO, W., HENCH, L.L., "Bioactive materials”, Ceramics International, v. 22, pp. 493-507, 1996.

[6] DUGGAN, D.M., JONHSON, B.L., "Dosimetry of the I-Plant Model 3500 iodine-125 brachytherapy source", Medical Physics, v. 28, n. 4, pp. 661-70, 2001. 
[7] HEPEL, J.T., SYED, A.M.N., PUTHAWALA, A., et al, "Salvage high-dose-rate brachytherapy for recurrent head-and-neck cancer", International Journal of Radiation Oncology Biology Physics, v. 62, n. 5, pp. 1444-1450, 2005.

[8] INSTITUTO NACIONAL DE CÂNCER, Ministério da Saúde, Estimativa 2006. Incidência de Câncer no Brasil, http://www.inca.gov.br/estimativa/2006/versaofinal.pdf, Acessado em março de 2006.

[9] MENDES, B.M., CAMPOS, T.P.R., "Investigation of the specific energy deposition from radionuclidehydroxyapatite macroaggregate in brain interstitial implants", Bioceramics, 2003.

[10] MENDES, B.M., CAMPOS, T.P.R., SILVA, C.H.T., "Radiological response of macroaggregates implants in an in-vitro experimental model", Bioceramics, 2003.

[11] MERCHANT, T.E., KIEHNA, E.N., LI, C., et al, "Radiation Dosimetry Predicts IQ After Conformal Radiation Therapy in Pediatric Patients with Localized Ependymona", International Journal of Radiation Oncology Biology Physics, v. 63, n. 5, pp. 1546-1554. 2005.

[12] PARKIN, D.M., PISANI, P., FERLAY, J., "Global cancer statistics", CA: A Cancer Journal for Clinicians, v. 49, n. 1, pp. 33-64, Jan-Feb 1999.

[13] ROBERTO, W.S., PEREIRA, M.M., CAMPOS, T.P.R., "Dosimetric analysis and characterization of radioactive seeds produced by the sol-gel method", Key Engineering Materials, vol. 240-242, pp. 579-582, 2002.

[14] ROBERTO, W.S., PEREIRA, M.M., CAMPOS, T.P.R., "Caracterização de vidros bioativos para tratamento de câncer de próstata”, In: $2^{\circ}$ Congresso Latino Americano de Orgãos Artificiais $e$ Biomateriais, Publicação em CD-Rom, Belo Horizonte, Dez. 2001.

[15] RIDGE, J.A., "Squamous cancer of the head and neck: Surgical treatment of local and regional recurrence", Seminars in Oncology, v. 20, pp. 419-429, 1993.

[16] SCHOTZIG, U., et al, "Standardization and decay data of Sm-153", Applied Radiation and Isotopes, v. 51, pp. 169-175, 1999.

[17] SILVA, G.X.O., CAMPOS, T.P.R., SIQUEIRA, S.L., MACIEL, M.B., "The Surgical Viability and Radiological Monitoring of Brain Implants of Biactive Micro-seeds in Animal Model”, Brasilian Archives of Biology and Technology an International Journal, v. 48, pp. 109-113, 2005.

[18] SIQUEIRA, M.G., NOVAES, V., Tumores intracranianos, Porto Alegre, Missau, 1982.

[19] SOWARDS, K.T., MEIGOONI, A.S., “A Monte Carlo evaluation of the dosimetric characteristics of the EchoSeed MM Model 6733 125I brachytherapy source”, Brachytherapy, v. 1, pp. 227-232, 2002.

[20] USGAONKER, S.R., MCNP Modeling of Prostate Brachytherapy and Organ Dosimetry, Tese de Mestrado, Texas A\&M University, Texas, USA, 2003. 